\title{
Sistema Único de Saúde (SUS): quando vai começar?
}

\author{
Unified Health System (UHS): when will it start?
}

\author{
Ana Lúcia Couto Coronel', Andrea Wander Bonamigo², Marcelo Schenck de \\ Azambuja², Helena Terezinha Hubert Silva² \\ 'Programa de Pós-graduação Ensino na Saúde, Universidade Federal de Ciências da Saúde de Porto Alegre, \\ Porto Alegre (RS), Brasil. \\ ${ }^{2}$ Universidade Federal de Ciências da Saúde de Porto Alegre, Porto Alegre, RS, Brasil.
}

Recebido: Dez. 16, 2016 Aceito: Mar. 20, 2017

\section{COMO CITAR ESTE ARTIGO}

Azambuja MS, et al. Sistema Único de

Saúde (SUS): quando vai começar?

Saúde (SUS): quando vai começar?
Interdisciplinary Journal of Health

Education. 2016 Ago-Dez;1(2):83-90

http://dx.doi.org/10.4322/ijhe.2016.016

\section{CORRESPONDÊNCIA}

Ana Lúcia Couto Coronel

Programa de pós-graduação Ensino na Saúde, Universidade Federal de Ciências da Saúde de Porto Alegre. Rua Sarmento Leite, 245, CEP 90050-170, Porto Alegre (RS), Brasil alc.coronel@hotmail.com

\section{FONTE DE FINANCIAMENTO}

Nenhuma

CONFLITO DE INTERESSE

Os autores declaram não haver

conflitos de interesse

O estudo foi realizado na Universidade Federal de Ciências da Saúde de Porto Alegre, Porto Alegre (RS), Brasil.

Todos os autores leram e aprovam a versão final submetida ao Interdisciplinary Journal of Health Education (IJHE).

\section{RESUMO}

O Sistema Único de Saúde (SUS) é um sistema em construção e conhecê-lo é fundamental para quem se dedica à assistência e ao Ensino Na Saúde. Este estudo, tem por objetivo revisar os marcos históricos da criação do Sistema Único de Saúde do Brasil, seus princípios e diretrizes, o perfil da utilização do sistema, procura identificar suas principais lacunas e, finalmente, contribuir através do relato de uma experiência profissional. É um artigo teóricoreflexivo, construído a partir da vivência dos autores na assistência, no ensino na saúde e da leitura de publicações pertinentes à área. A partir da revisão realizada, foi possível concluir que, passadas quase três décadas da sua criação, persiste a busca pela consolidação das diretrizes do SUS, que a sua utilização pela população está crescendo, que há falta de recursos humanos e materiais, assim como o uso inadequado dos recursos disponíveis. Contudo, são sugeridas práticas capazes de minimizar as lacunas encontradas e pesquisas que possam enriquecer e apontar novas propostas.

PAlavras-chave: Sistema Único de Saúde. Sistemas de Saúde. Assistência à saúde.

\section{ABSTRACT}

The Unified Health System (UHS) is a system under construction and knowing it is crucial for those who dedicate themselves to assistance and Health Education. This study aims to review the historical milestones of the creation of the Unified Health System of Brazil; its principles and guidelines; the profile of the use of the system, seeks to identify its main gaps and, finally, contribute through the report of a professional experience. It is a theoretical-reflective article, built from the experience of the authors in the assistance, teaching in health and reading publications relevant to the area. Based on the review, it was possible to conclude that, after almost three decades of its creation, the search for consolidation of UHS guidelines persists, that its use by the population is growing, that there is a lack of human and material resources, as well as the misuse of available resources. However, practices are suggested that can minimize the gaps found and research that can enrich and point out new proposals.

KEYWORDS: Unified Health Systems. Health Systems. Delivery of health care. 


\section{Introdução}

"Mas quando é que vai começar?"

Essa interrogação, fruto da inquietação frente a tantas dúvidas e sugestões, foi proferida pela docente, durante uma aula no mestrado Ensino na Saúde, na Universidade Federal de Ciências da Saúde de Porto Alegre. A turma debatia temas conhecidos na área: objetivos, potencialidades e dificuldades no Sistema Único de Saúde (SUS), com os alunos focados no último aspecto citado. A questão levantada, prática em sua essência, revelou a expectativa existente quanto à consolidação dos propósitos para a saúde pública no Brasil e direcionou esta busca para um melhor entendimento desse sistema tão complexo. Conhecer e entender a dinâmica da saúde pública brasileira é parte indispensável àqueles que nela atuam, principalmente quando o desempenho profissional tem foco no ensino. O reconhecimento da importância da integração entre ensino, atenção, gestão e controle social não é novo e necessita ser aplicado na prática ${ }^{1}$. Para isso, entendemos que o conhecimento da história, é capaz de motivar uma busca por melhores resultados, uma vez que ensina através das experiências prévias. O entendimento dos alicerces do SUS, pode direcionar essa busca de forma coordenada, a fim de concretizar os objetivos do sistema. Analisar o comportamento da população, conhecendo suas necessidades e insatisfações, dá sentido e perspectivas aos projetos e ações propostos. Reconhecer as falhas nas engrenagens entre os setores envolvidos, permite refletir e atuar nas soluções necessárias e a experiência profissional na assistência, traz para a realidade a teoria desenvolvida. Assim, apresentaremos alguns marcos históricos da saúde pública no Brasil e da criação do SUS, seus princípios e diretrizes, o perfil de utilização do sistema, o apontamento de suas principais lacunas e um relato da experiência de um dos autores, enquanto atuante na área assistencial.

\section{Marcos históricos}

A história da saúde pública no Brasil, que teve seu início na primeira década do século XIX, tem sido amplamente apresentada e analisada em diversos estudos ${ }^{2-5}$. Inicialmente, Emílio Ribas em São Paulo e Osvaldo Cruz no Rio de Janeiro, introduziram a teoria bacteriológica para intervenções no processo saúde-doença, sendo esse processo entendido como o resultado da exposição do ser humano aos efeitos insalubres do meio. A partir daí, inspirado no modelo norte-americano de saúde pública, surgiu o movimento médico-sanitarista, que buscava o saneamento como meio de garantir a saúde coletiva e responsabilizava o indivíduo para alcançar esse objetivo. Nesse sentido, diversas práticas campanhistas foram sendo desenvolvidas de forma vertical, sob a coordenação do Estado e visando, principalmente, o saneamento da zona rural, considerada fundamental para a interiorização e expansão do país. As campanhas sanitárias foram criadas para combate de problemas específicos, como malária, febre amarela e saúde das crianças. Postos e centros de saúde, coordenados por sanitaristas com dedicação integral, foram inaugurados. Durante esse período, a assistência médica era tida como pouco eficaz e estava dissociada da prevenção. Em 1953, foi criado o Ministério da Saúde e em 1956 o Departamento de Endemias Rurais, que mantiveram o mesmo modelo nas intervenções.

O modelo médico-sanitarista, no entanto, mostrou-se incapaz de conter a miséria e a degradação do estado de saúde da população e seguiu-se a ele o movimento centrado na perspectiva do desenvolvimento, que buscava ações coordenadas de saúde. Nesse momento, o desenvolvimento econômico era visto como condição indispensável para o alcance da saúde coletiva. Porém, a crise econômica e política, que culminou em 1964, desviou os rumos do gerenciamento da saúde e este passou a ter um enfoque assistencial e mercadológico, com financiamento e expansão do setor privado. O país apresentava um sistema dividido em "medicina previdenciária" e "saúde pública", 
estando o primeiro a cargo dos institutos de pensões e voltado aos trabalhadores formais das zonas urbanas, enquanto o segundo, era direcionado às zonas rurais e esferas mais pobres da população, com ações, essencialmente, preventivas. A falta de organização do sistema, entretanto, gerou a necessidade de maior racionalização das ações e originou programas para redesenhar a administração da saúde pública, voltando os olhos para a municipalização da coordenação. Os movimentos sociais e políticos refletiam a insatisfação popular e, nesse contexto, a saúde apresentava um quadro preocupante de baixa cobertura assistencial e disseminação de doenças infecto-parasitárias, típicas da pobreza. O movimento de reforma sanitária (MRS) surgiu em meio às manifestações de oposição ao regime político, difundindo ideias de expansão da assistência, com foco na atenção primária, organizada de forma hierárquica e regionalizada, em consonância com orientações da Organização Mundial de Saúde (OMS) e com a Organização Pan-Americana de Saúde (OPAS). Uma das suas inspirações, foi o National Health System (NHS) da Inglaterra, criado em 1948, a partir do Relatório Dawson, que apresentava o ideário da integração entre a medicina assistencial e preventiva, com a distribuição em rede do sistema de saúde e centrado na assistência básica ${ }^{6}$. Assim, através de ações como a criação do Centro Brasileiro de Estudos de Saúde (Cebes), veiculação do periódico Saúde em Debate, criação da Associação Brasileira de Saúde Coletiva (Abrasco) e, entre outras, a realização do primeiro Simpósio de Política Nacional de Saúde, a reforma sanitária brasileira (RSB) buscou consolidar-se 7 . Em 1986, a $8^{\text {a }}$ Conferência Nacional de Saúde aprovou o que representaria as bases da reforma sanitária:

O direito universal à saúde; o caráter intersetorial dos determinantes da saúde; o papel regulador do Estado em relação ao mercado de saúde; a descentralização, regionalização e hierarquização do sistema; a participação popular; o controle democrático e, fundamentalmente, a necessidade de integração entre saúde previdenciária e saúde pública ${ }^{8}$.

Em 1988, a Constituição Federal estabelece que a saúde é direito de todos e dever do Estado e é criado o Sistema Único de Saúde, cuja implementação teve início em 1990.

\section{Principios e diretrizes do SUS}

O Sistema Único de Saúde apresentou-se ambicioso em um país marcado pelas desigualdades sociais, tendo por princípios a universalidade, a integralidade e a equidade no atendimento ${ }^{10}$. Estruturou-se de modo descentralizado, regionalizado, hierarquizado e democrático. O controle social, com a população participando das decisões referentes à gestão da saúde e assegurando seus interesses, os conselhos de saúde e os comitês intergestores, mantém o caráter democrático do SUS ${ }^{11-13}$. É único, porque segue os mesmos princípios em todo o território nacional, sendo o município o maior responsável pela gestão do atendimento e por desenvolver ações de promoção e proteção à saúde ${ }^{14}$. É universal, pois destina-se a toda a população, sem distinção e é integral, porque busca olhar o indivíduo em toda a sua complexidade, prestando todo o atendimento necessário para a obtenção e manutenção da saúde, sendo equânime nessa prestação. Organizado em redes de atenção, distribuídas em diferentes graus de complexidade, tem na atenção básica ou primária, a "porta de entrada" do sistema. No nível primário, devem ocorrer as medidas preventivas, o controle de agravos à saúde, a identificação e encaminhamento de casos que necessitem serviços mais complexos. A exemplo dos serviços primários, estão as unidades de Estratégia de Saúde da Família (ESF), as Unidades Básicas de Saúde (UBS), programas como o Programa Agentes Comunitários de Saúde (PACS) ou o Programa Brasil Sorridente, de saúde bucal ${ }^{15}$. Em níveis de maior complexidade, estão os serviços especializados, os hospitais, laboratórios e exames de alto custo, que devem ser acionados quando os problemas não forem solucionados na atenção básica. 
Os países que adotaram sistemas semelhantes, como os países nórdicos e o Canadá, fizeram adequações às suas realidades, mas sempre tentando manter a premissa de que a rede básica deve ser resolutiva, com a qualificação dos recursos humanos, com acesso aos meios diagnósticos e terapêuticos e com interação entre os componentes da rede ${ }^{6}$. Com quase trinta anos, o SUS cresceu, amadureceu, mas ainda é alvo de discussões e críticas, tanto em nível acadêmico, quanto em meio à própria população, que se diz insatisfeita quanto à acessibilidade, coordenação, integração de cuidados, serviços prestados e orientação dispensada pelos responsáveis pela assistência ${ }^{16}$.

\section{Utilização do suS}

Em um sistema de saúde universal, integral e equânime, deve haver garantia na prestação de serviços em todos os níveis de complexidade e com a demanda tecnológica necessária para garantir resolubilidade. A porta de entrada do SUS, como já referido anteriormente, é a atenção básica. Nela, o indivíduo recebe consulta médica e de enfermagem, atendimento psicológico, nutricional, odontológico e social, entre outras áreas profissionais. Recebe verificação de sinais vitais, nebulizações, curativos, vacinação, aplicação de medicamentos injetáveis, participa de programas e atendimentos em grupos, recebe medicamentos da farmácia básica e, quando necessário, é encaminhado a centros especializados ${ }^{17}$. Nesse caso, são utilizados documentos de referência e contra referência, criados para vincular os profissionais de todos os níveis de atenção, através da informação clínica. Uma vez tendo feito exames, consultas ou procedimentos especializados, o indivíduo deve retornar ao nível de atenção primária para acompanhamento.

A utilização do SUS vêm apresentando crescimento, tendo sido de 49,3\% em 1998, 56,5\% em 2003 e 57,2\% em 2008 ${ }^{18}$. O setor privado, entretanto, detém a maior concentração de hospitais e leitos para internação, assim como de serviços de apoio diagnóstico e terapêutico (SADT), enquanto o serviço público detém o maior número de unidades ambulatoriais (excluídos os consultórios privados) ${ }^{19}$. Quanto ao perfil sociodemográfico dos usuários do SUS, estudos demonstraram, que há uma maior utilização dos serviços por indivíduos com menor renda e escolaridade, portadores de doenças crônicas, havendo predominância de mulheres e a maioria refere buscar atendimento por motivo de doença, sendo crescente a procura devido à acidente ${ }^{20-22}$. Segundo dados do suplemento de saúde da Pesquisa Nacional por Amostra de Domicílios (PNAD), usuários de serviços privados, com melhor condição socioeconômica, fazem uma maior utilização dos serviços de saúde com vistas à prevenção do que indivíduos com baixa renda. Cerca de $4 \%$ informa não ter sido atendido ao buscar serviços de saúde e quanto aos motivos para falta de atendimento, foram referidos: falta de vaga, falta de médico ou de especialista, espera prolongada, falta de funcionamento de serviços ou equipamento e incapacidade de pagamento ${ }^{20}$.

\section{Lacunas do Sistema}

Entre as principais dificuldades do Sistema Único de Saúde, são apontadas: a falta de médicos, formação inadequada dos profissionais da saúde, falta de integração entre os componentes da rede assistencial e o subfinanciamento da saúde. Tanto a avaliação inicial, quanto o seguimento, são apontados pelos usuários como de difícil acesso e os gestores, referem um excesso de encaminhamentos aos especialistas ${ }^{23}$.

A OMS e a OPAS, não fazem recomendação quanto ao número de médico por habitante, embora tenha sido a elas atribuída a recomendação de 1 médico para cada 1000 habitantes, como sendo o ideal a um bom sistema de saúde ${ }^{24}$. O Conselho Federal de Medicina (CFM) informa que existem atualmente, 417.801 médicos registrados no Brasil, sendo que, 314.422 concentram-se nos estados do sul e sudeste ${ }^{25}$. O Censo de 
2010, calculou uma população de 190.732 .694 habitantes no país ${ }^{26}$, o que significa que há cerca de 1 médico para cada 456,52 habitantes no total. Porém, se for calculada por região, essa média se altera significativamente. Por exemplo, para uma população estimada de 803.513 habitantes no Acre em 2015, havia 895 médicos registrados, totalizando uma média de 1 médico para cada 897,78 habitantes, enquanto em São Paulo, essa média cai para 1 médico por 354,93 habitantes. Contudo, tomada como único indicador, a análise do "número de médicos por habitante", pode falsear a interpretação das reais necessidades da população, pois há muitos outros fatores envolvidos na dinâmica assistencial. Nesse viés, muitos programas e políticas públicas se apoiaram, sem conseguir resolver a questão da dificuldade de acesso aos serviços de saúde. Fontes estatais apresentam dados aparentemente satisfatórios quanto ao desempenho do SUS:

Brasil é o único país do mundo com mais de 100 milhões de habitantes que tem um sistema de saúde público e gratuito. Somente em 2014, o sistema contabilizou 4,1 bilhões de tratamentos ambulatoriais, 1,4 bilhão de consultas médicas e 11,5 milhões de internações. No ano passado, o total de recursos investidos em ações e serviços públicos de saúde foi de $\mathrm{R} \$ 92,2$ bilhões e este ano será R \$ 98,4 bilhões. Os valores financiam programas, como Saúde da Família, que hoje atinge a 112 milhões de habitantes, ou seja, mais da metade da população brasileira (56\%) ${ }^{27}$.

Possivelmente, portanto, não seja apenas uma questão de números. A formação de profissionais para trabalhar no SUS, tem sido apontada como um dos "nós críticos" do sistema e o modelo flexneriano do ensino na saúde, como ultrapassado e inadequado ${ }^{28}$. Postula-se, que haja uma maior inserção do estudante de graduação ou mesmo do profissional em processo continuado de aprendizado, dentro da rede assistencial do Sistema Único de Saúde, para que conheçam seus mecanismos e desenvolvam uma postura adequada às suas propostas ${ }^{29}$. Sabe-se, entretanto, que isso já acontece nas escolas médicas e em outras áreas da saúde, onde graduandos e residentes têm sua formação em hospitais escola e ambulatórios voltados ao atendimento do SUS. A classe médica, por sua vez, defende que a valorização dos profissionais da saúde, através da adoção de planos de cargos, carreira e vencimentos e a criação de uma carreira de Estado, poderiam estimular o interesse pelo serviço público e garantir uma distribuição mais uniforme desses profissionais ${ }^{24}$. $\mathrm{O}$ crescimento dos serviços especializados, serviços de diagnóstico e tratamento e leitos hospitalares, não acompanhou o crescimento das Unidades Básicas de Saúde e unidades de Estratégia da Saúde da Família, criando uma demanda reprimida em diversos setores. Isso remete a outro "nó crítico": o financiamento da saúde no país. O SUS é financiado pelas receitas estatais e de contribuições sociais dos orçamentos federal, estadual e municipal e os recursos têm sido insuficientes para garantir o funcionamento adequado dos serviços, acumulando um déficit significativo nas instituições ${ }^{30}$. Embora tenha sido definido pelos constituintes, que alíquotas das contribuições sociais e dos recursos governamentais, deveriam ser destinados unicamente à Seguridade Social, isso nunca foi cumprido e mesmo os recursos captados pela Contribuição Provisória sobre Movimentações Financeiras (CPMF), criada para financiar a saúde, foram desviados para outros fins ${ }^{31}$.

\section{Experiência na assistência: relato de um dos autores*}

Em 2006, após concurso público municipal, fui nomeada para integrar uma Unidade Básica de Saúde, apesar de ser médica especialista, com formação cirúrgica. Não recebi nenhuma capacitação quanto aos mecanismos de funcionamento da rede assistencial, apenas informações quanto ao preenchimento de alguns formulários

\footnotetext{
* Médica Coloproctologista em um hospital municipal de Porto Alegre, RS, Brasil.
} 
e, assim, iniciei o atendimento. Havia um grande número de pessoas aguardando atendimento na minha área e o tempo determinado para cada atendimento foi de 15 minutos, o mesmo das demais áreas. Os exames e cirurgias eram encaminhados aos serviços de maior complexidade. Logo, à medida que ocorriam mais atendimentos, a demanda por esses procedimentos aumentava, sem que houvesse capacidade para sua absorção nos demais níveis. O escasso tempo para cada atendimento e a falta de recursos, limitavam a resolução de todas as questões trazidas nas consultas, apesar de que muitas dessas questões, estivessem relacionadas a falta de orientação dietética ou fossem secundários a doenças crônicas descompensadas, como diabetes e obesidade. Em 15 minutos, o atendimento tinha que ser focado na queixa principal, o exame físico específico e sumário e as orientações objetivamente direcionadas à especialidade. Em 2010, fui transferida para um hospital, onde poderia realizar exames e cirurgias, na expectativa de aliviar a demanda reprimida. Entretanto, a falta de materiais, o déficit de pessoal e de equipamentos específicos, somavam-se à falta de recursos e à burocracia para a sua aquisição, mantendo a dificuldade de completar o atendimento iniciado na rede básica. Desse modo, ainda hoje, o número de pessoas que encaminho para procedimentos é maior do que o número de procedimentos que consigo realizar, alimentando a demanda reprimida. Embora esteja em um hospital, muitos dos indivíduos que atendo são portadores de distúrbios relacionados a má alimentação, como, por exemplo, a constipação intestinal e suas consequências ou doenças relacionadas à falta de educação preventiva, como as doenças sexualmente transmissíveis, questões que deveriam ser tratadas na atenção básica. Com isso, apesar dos esforços de triagem, pessoas com doenças potencialmente graves, esperam na fila de consultas e exames para obterem avaliação, diagnóstico e tratamento.

\section{Considerações finais}

Essa breve reflexão sobre o sistema de saúde pública brasileiro, apresenta limitações relacionadas à revisão não sistemática do tema e à visão dos autores com foco na assistência e na educação. Ainda assim, mostra que a busca pela consolidação dos princípios e diretrizes do SUS persiste e, apesar de muitas conquistas terem sido alcançadas, ainda há muito por fazer.

A solução dos problemas é ampla, passando pela administração pública, pelos gestores, pelos políticos, pelas escolas e universidades e pela sociedade em geral. Os governos, precisam olhar além dos números, buscando indicadores de qualidade e cumprindo as determinações constitucionais para o financiamento da saúde. Os gestores, devem investir mais e valorizar os recursos humanos, também indo além dos números, capacitando, sobretudo, a rede básica e aproveitando adequadamente a mão de obra qualificada. Os políticos, têm o dever de criar mecanismos facilitadores para a administração pública, desburocratizando e acelerando os processos de aquisição de recursos, em prol do bem-estar social. A sociedade, por sua vez, deve assumir a responsabilidade pela sua saúde, saindo da posição passiva de "ser cuidada", buscando seus direitos, participando no controle social e cumprindo seus deveres. As escolas devem educar, não só para a rotina acadêmica, mas para a cidadania, formando profissionais comprometidos com a sociedade. $\mathrm{O}$ ensino na saúde, especialmente, deve promover uma abordagem interdisciplinar e formativa (não apenas informativa) e disseminar a visão do indivíduo como o ser complexo e completo queé. Não um doente, um paciente ou um usuário do sistema, mas uma pessoa em busca de auxílio. A qualificação de profissionais, dos serviços, da oferta de serviços, a gestão do trabalho, o aprofundamento na integração ensino-serviço-comunidade, podem ser estimulados através da realização de pesquisas aplicadas e da devolução dos produtos e processos resultantes à comunidade. Por fim, a comunicação deve 
prevalecer entre todos os níveis, pois as boas práticas e políticas públicas eficientes, dependem da troca de informação entre todos os atores envolvidos.

As informações e análises aqui apresentadas, não têm a pretensão de esgotar o tema, mas almejam instigar e instrumentar o leitor para sua própria busca e reflexão.

\section{Referências}

1. Ceccim RB, Feuerwerker LCM. O quadrilátero da formação para a área da saúde: ensino, gestão, atenção e controle social. Physis. 2004;14(1):41-65. http://dx.doi.org/10.1590/S0103-73312004000100004.

2. Merhy EE, Queiroz MS. Saúde pública, rede básica e o sistema de saúde brasileiro. Cad Saude Publica. 1993;9(2):177-84. PMid:15448839. http://dx.doi.org/10.1590/S0102-311X1993000200009.

3. Viacava F, Almeida C, Caetano R, et al. Uma metodologia de avaliação do desempenho do sistema de saúde brasileiro. Ciênc Saúde Coletiva. 2004;9(3):711-24.

4. Fleury S. Reforma sanitária brasileira: dilemas entre o instituinte e o instituído. Cien Saude Colet. 2009;14(3):743-52. PMid:19547774. http://dx.doi.org/10.1590/S1413-81232009000300010.

5. Amarante P, Rizzotto MLF, Costa AM. The history of a movement: Saude em Debate magazine and Brazilian health reform. Cien Saude Colet. 2015;20(7):2023-9. PMid:26132241. http://dx.doi.org/10.1590/1413-81232015207.05752015.

6. Kuschnir R, Chorny AH. Redes de atenção à saúde: contextualizando o debate. Cien Saude Colet. 2010;15(5):2307-16. PMid:20802864. http://dx.doi.org/10.1590/S1413-81232010000500006.

7. Paiva CHA, Teixeira LA. Reforma sanitária e a criação do Sistema Único de Saúde: notas sobre contextos e autores. Hist Cienc Saude Manguinhos. 2014;21(1):15-35. PMid:24789484. http://dx.doi.org/10.1590/S0104-59702014000100002.

8. Paulus A Jr, Cordoni L Jr. Políticas públicas de saúde no Brasil. Espaço para a Saúde. 2006;8(1):13-19.

9. Brasil. Portal da Saúde. Do sanitarismo à municipalização. Brasília (DF): MS; 2017 [citado em 2017 Fev 12]. Disponível em: http://portalsaude.saude.gov.br/index.php/o-ministerio/historico.

10. Teixeira C. Os princípios do sistema único de saúde. Texto de apoio elaborado para subsidiar o debate nas Conferências Municipal e Estadual de Saúde Salvador, Bahia. 2011 [citado em 2017 Jun 01]. Disponível em: http://www.saude.ba.gov. br/pdf/os_principios_do_sus.pdf

11. Carvalho AI. Conselhos de saúde, responsabilidade pública e cidadania: a reforma sanitária como reforma do Estado. Saúde e democracia: a luta do CEBES. São Paulo (SP): Lemos Editorial; 1997. p. 93-112.

12. Correia MVC. Que controle social. Os conselhos de saúde como instrumento. Rio de Janeiro (RJ): Fiocruz; 2000.

13. Tatagiba L. Os conselhos gestores e a democratização das políticas públicas no Brasil. Sociedade civil e espaços públicos no Brasil. São Paulo (SP): Paz e Terra; 2002. p. 47-103.

14. Brasil. Ministério da Saúde. ABC do SUS: doutrinas e princípios. Brasília (DF): MS; 2017 [citado em 2017 Jun 01]. Disponível em: http://www.pbh.gov.br/smsa/bibliografia/abc_do_sus_doutrinas_e_principios.pdf

15. Fundação Oswaldo Cruz (FIOCRUZ). Pense SUS. Rio de Janeiro (RJ): FIOCRUZ; 2017 [citado em 2017 Fev 13]. Disponível em: http://www.pensesus.fiocruz.br.

16. Oliva ACD, Moura CMR, Lima CA, Costa FM, Rocha JFD. Avaliação dos atributos do cuidado primário de saúde na perspectiva do usuário. Revista Uniabeu. 2015;8(18):196-208.

17. Schwartz TD, Ferreira JTB, Maciel ELN, Lima RCD. Estratégia Saúde da Família: avaliando o acesso ao SUS a partir da percepção dos usuários da Unidade de Saúde de Resistência, na região de São Pedro, no município de Vitória (ES). Ciênc Saúde Coletiva. 2010;15(4):2145-54.

18. Dilélio AS, Tomasi E, Thumé E, et al. Padrões de utilização de atendimento médico-ambulatorial no Brasil entre usuários do Sistema Único de Saúde, da saúde suplementar e de serviços privados. Cad Saude Publica. 2014;30(12):2594-606. PMid:26247989. http://dx.doi.org/10.1590/0102-311X00118713.

19. Santos IS, Ugá MAD, Porto SM. O mix público-privado no Sistema de Saúde Brasileiro: financiamento, oferta e utilização de serviços de saúde. Cien Saude Colet. 2008;13(5):1431-40. PMid:18813646. http://dx.doi.org/10.1590/S1413-81232008000500009.

20. Ribeiro MCSA, Barata RB, Almeida MF, Silva ZP. Perfil sociodemográfico e padrão de utilização de serviços de saúde para usuários e não-usuários do SUS-PNAD 2003. Cien Saude Colet. 2006;11(4):1011-22. http://dx.doi.org/10.1590/ S1413-81232006000400022.

21. Silva ZP, Ribeiro MCSA, Barata RB, Almeida MF. Perfil sociodemografico e padrao de utilizacao dos servicos de saude do Sistema Unico de Saude (SUS), 2003-2008. Cien Saude Colet. 2011;16(9):3807-16. PMid:21987323. http://dx.doi. org/10.1590/S1413-81232011001000016.

22. Fernandes LCL, Bertoldi AD, Barros AJD. Utilização dos serviços de saúde pela população coberta pela Estratégia de Saúde da Família. Rev Saude Publica. 2009;43(4):595-603. PMid:19547801. http://dx.doi.org/10.1590/S0034-89102009005000040.

23. Azevedo ALM, Costa AM. A estreita porta de entrada do Sistema Único de Saúde (SUS): uma avaliação do acesso na Estratégia de Saúde da Família. Interface-Comunicação, Saúde, Educação. 2010;14(35):797-810.

24. Scheffer M, Biancarelli A, Cassenote A. Demografia médica no Brasil: dados gerais e descrições de desigualdades. São Paulo (SP): Conselho Regional de Medicina do Estado de São Paulo, Conselho Federal de Medicina. 2011. p. 1.

25. Conselho Federal de Medicina (CFM). Brasília (DF): CFM; 2017 [citado em 2017 Fev 13]. Disponível em: http://portal. cfm.org.br/?option=com_estatistica.

26. Instituto Brasileiro de Geografia e Estatística (IBGE). Rio de Janeiro (RJ): IBGE; 2017 [citado em 2017 Fev 13]. Disponível em: http://www.ibge.gov.br/home/estatistica/populacao/censo2010/default.shtm 


\section{ijhe}

27. Brasil. Ministério da Saúde. Portal Brasil. Brasília (DF): MS; 2016 [citado em 2017 Jun 01]. Disponível em: http://www. brasil.gov.br/saude/2015/06/populacao-teve-acesso-a-1-4-bi-de-consultas-medicas-pelo-sus-em-um-ano

28. Lampert JB. Tendências de mudanças na formaçäo médica no Brasil: tipologia das escolas. São Paulo (SP): Hucitec; 2002.

29. Araújo D, Miranda MCG, Brasil SL. Formação de profissionais de saúde na perspectiva da integralidade. Revista Baiana de Saúde Pública. 2014;31:20.

30. Paim JS, Teixeira CF. Configuração institucional e gestão do Sistema Único de Saúde: problemas e desafios. Cien Saude Colet. 2007;12(Supl):1819-29. http://dx.doi.org/10.1590/S1413-81232007000700005.

31. Mendes Á, Marques RM. O financiamento do SUS sob os "ventos" da financeirização. Cien Saude Colet. 2009;3(3):841-50. PMid:19547783. http://dx.doi.org/10.1590/S1413-81232009000300019.

\section{Contribuição dos autores}

Ana Lúcia Couto Coronel contribuiu substancialmente para a concepção e planejamento do estudo, aquisição, análise e interpretação dos dados do estudo. Andrea Wander Bonamigo contribuiu para a concepção e planejamento do estudo e aprovação final da versão submetida. Marcelo Schenck de Azambuja participou da aprovação final da versão submetida e esteve disponível para todos os aspectos do trabalho, assegurando que questões relacionadas à acurácia e integridade de qualquer parte do trabalho. Helena Terezinha Hubert Silva participou da aprovação final da versão submetida e esteve disponível para todos os aspectos do trabalho, assegurando que questões relacionadas à acurácia e integridade de qualquer parte do trabalho. 